\title{
Huaier increases the antitumor effect of gemcitabine on pancreatic cancer in vitro and in vivo
}

\author{
Tao Chen ${ }^{1 \#}$, Dongbao Li ${ }^{1,2,3 \#}$, Chao Feng ${ }^{4 \#}$, Zixiang Zhang ${ }^{1}$, Dongming Zhu ${ }^{1}$, Dechun Li ${ }^{1}$, Xin Zhao ${ }^{1,2,3}$ \\ ${ }^{1}$ Department of General Surgery, The First Affiliated Hospital of Soochow University, Suzhou, China; ${ }^{2}$ Jiangsu Key Laboratory of Gastrointestinal \\ Tumor Immunology, The First Affiliated Hospital of Soochow University, Suzhou, China; ${ }^{3}$ Jiangsu Key Laboratory of Clinical Immunology, \\ Soochow University, Suzhou, China; ${ }^{4}$ Soochow University Institutes for Translational Medicine, Suzhou, China \\ Contributions: (I) Conception and design: T Chen, X Zhao; (II) Administrative support: D Li, D Li, X Zhao; (III) Provision of study materials or \\ patients: C Feng, Z Zhang, D Zhu; (IV) Collection and assembly of data: T Chen, D Li, C Feng; (V) Data analysis and interpretation: T Chen, D Li, \\ X Zhao; (VI) Manuscript writing: All authors; (VII) Final approval of manuscript: All authors. \\ \#These authors contributed equally to this work. \\ Correspondence to: Xin Zhao. Department of General Surgery, The First Affiliated Hospital of Soochow University, No. 188 Shizi Street, Suzhou \\ 215006, China. Email: zhaox@suda.edu.cn.
}

Background: Pancreatic cancer has a high degree of malignancy and poor prognosis. As the first-line chemotherapy drug for pancreatic cancer, gemcitabine is widely used but is limited in its efficacy due to the development of chemoresistance. Huaier is a traditional Chinese medicine with anticancer effects. This present study explored the antitumor effect of gemcitabine combined with Huaier on pancreatic cancer in vitro and in vivo.

Methods: After treatment with gemcitabine combined with Huaier in PaTu8988 pancreatic cancer cells, including 3-(4,5-dimethyl-2-thiazolyl)-2,5-diphenyl-2-H-tetrazolium bromide (MTT) assay, wound healing, and Transwell invasion in vitro assays were performed to investigate the proliferation, migration and invasion of cells, respectively. The apoptotic rate of cells was detected by propidium iodide_-annexin V staining and flow cytometry. In vivo PaTu8988 pancreatic cancer xenograft and tail vein injection into lung metastasis nude mice models were used to determine the tumor growth and lung metastasis efficiency.

Results: Huaier could not only inhibit the proliferation, migration, and invasion of cancer cells, but could also induce the apoptosis of pancreatic cancer in vitro and suppress tumor growth and lung metastasis in vivo. It further significantly increased the tumor suppressing effects of gemcitabine, and combined use of the two drugs exhibited a synergistic effect.

Conclusions: Our present study concluded that Huaier was capable of enhancing the antitumor effect of gemcitabine in pancreatic cancer in vitro and in vivo. Therefore, Huaier may be a potential drug to increase the therapy sensitivity of gemcitabine and improve the prognosis of pancreatic cancer patients.

Keywords: Huaier; pancreatic cancer; gemcitabine; antitumor; traditional Chinese medicine (TCM)

Submitted Jul 30, 2020. Accepted for publication Jan 15, 2021.

doi: $10.21037 /$ tcr-20-2627

View this article at: http://dx.doi.org/10.21037/tcr-20-2627

\section{Introduction}

Pancreatic cancer is one of the most aggressive cancer types and the fourth most lethal malignancy worldwide (1). Based on the most recent statistics from the American Cancer Society (ACS), 57,600 new pancreatic cancer cases and 47,050 deaths are estimated to occur in the United
States in 2020, indicating almost equivalent incidence and mortality rates (1). A lack of early symptoms means most patients are diagnosed at advanced stages of the disease reducing the likelihood of successful surgical intervention (2). Moreover, advanced pancreatic cancer patients respond poorly to chemotherapy and radiotherapy (3). Thus the 
5 -year survival rate of pancreatic cancer is still the lowest among cancers at approximately 9\% (1). The nucleoside analog, gemcitabine, has been the cornerstone of adjuvant chemotherapy in pancreatic cancer treatment for decades (4). However, gemcitabine resistance develops within a few weeks of initiation of chemotherapy (5), and it is necessary to explore novel drugs to overcome the resistance and improve sensitivity to gemcitabine.

Traditional Chinese medicine (TCM) has been used for thousands of years, and has been reported to reduce inflammation and treat some diseases, such as malaria. Trametes robiniophila Murr, commonly known as Huaier, has been used as a TCM therapy for approximately 1,600 years (6). The most effective extract of Huaier is proteoglycan, which is composed of polysaccharides, amino acids, and water, among which the polysaccharide component has been proven to be the main active ingredient responsible for its therapeutic effects (7). In recent years, the anticancer effects of Huaier have begun to attract the attention of researchers, with an increasing number of studies having demonstrated its efficacy in suppressing the progression of various tumors such as breast cancer (8-10), hepatocellular carcinoma (11-13), lung cancer $(14,15)$ and renal cancer $(16)$. The anticancer mechanisms of Huaier including proliferation inhibition, anti-metastasis, anti-angiogenesis, apoptosis induction, tumor-specific immunomodulatory and cancer stem cell inhibitory activities have also been validated (6).

A recent study has also shown that Huaier extract could restrain the proliferation and invasion of pancreatic cancer cells (17). However, few studies have focused on the effect of Huaier combined with chemotherapy for cancer treatment, including the combination of gemcitabine and Huaier. Therefore, in this present study, we detected the proliferation, apoptosis activity, migration and invasion of pancreatic cancer cells treated with gemcitabine combined with Huaier in vitro. We also established nude mice xenograft and lung metastasis models to explore whether Huaier could enhance the antitumor effect of gemcitabine on pancreatic cancer in vivo. We present the following article in accordance with the ARRIVE reporting checklist (available at: http://dx.doi.org/10.21037/tcr-20-2627).

\section{Methods}

\section{Reagents}

We purchased RPMI 1640 and fetal bovine serum (FBS) from Hyclone (Logan, UT, USA), and electuary ointment of Huaier was purchased from Gaitianli Medicine Co. Ltd (Jiangsu, China). The electuary ointment of Huaier (5 g) was dissolved in $50 \mathrm{~mL}$ of complete medium, and sterilized with $0.22 \mu \mathrm{m}$ of filter to obtain a $100 \mathrm{mg} / \mathrm{mL}$ stock solution for long-term storage at $-20^{\circ} \mathrm{C}$, and diluted to obtain $1.5,3$, 6,9 and $12 \mathrm{mg} / \mathrm{mL}$ work solutions before administration of in vitro assays. For in vivo experiments, Huaier extract was freshly prepared (50 $\mathrm{mg}$ dissolved in $0.1 \mathrm{ml}$ sterile normal). Gemcitabine was purchased from Selleck Chemicals (Shanghai, China), $200 \mathrm{mg}$ of which was dissolved in 6.7 $\mathrm{mL}$ of sterile normal saline to obtain the $0.1 \mathrm{~mol} / \mathrm{ml}$ stock solution for long-term storage at $-80^{\circ} \mathrm{C}$.

\section{Cell line and cell culture}

The pancreatic cancer cell line, PaTu8988, was purchased from the cell bank of Chinese Academy of Science (Shanghai, China). Cells were cultured in RPMI 1640 supplemented with 10\% FBS and 1\% penicillin-streptomycin (Beyotime, Beijing, China) under $5 \% \mathrm{CO}_{2}$ at $37^{\circ} \mathrm{C}$.

\section{Cell viability MTT assay}

The untreated control group (complete medium), gemcitabine groups $(2,4,8,12$ and $16 \mu \mathrm{M})$, Huaier groups $(1.5,3.0,6.0,9.0$ and $12.0 \mathrm{mg} / \mathrm{mL}$ of terminal concentrations), and gemcitabine $(8 \mu \mathrm{M})$ plus Huaier $(6 \mathrm{mg} / \mathrm{mL})$ (the dose of gemcitabine or Huaier that could inhibit approximately 50\% cell viability in PaTu8988 cells) groups were prepared. Then, 3-(4,5-dimethyl-2-thiazolyl)2,5-diphenyl-2-H-tetrazolium bromide assay (MTT; Sigma, Shanghai, China) assays were performed to estimate the viability of $\mathrm{PaTu} 8988$ cells in the groups. A total of 5,000 cells/well were plated into 96-well plates for $24 \mathrm{~h}$ and treated with gemcitabine, Huaier, or the combination of the two drugs for an additional $72 \mathrm{~h}$. MTT $(50 \mu \mathrm{L})$ was added to cells at 24,48 and $72 \mathrm{~h}$ after treatment and incubated for $4 \mathrm{~h}$ at $37^{\circ} \mathrm{C}$ in a dark environment, with $200 \mu \mathrm{L}$ dimethyl sulfoxide (DMSO) being subsequently added. Finally, the optical density (OD) was read at an absorbance of $490 \mathrm{~nm}$, and the percentage of cells in each group was calculated relative to the untreated control group. Experiments were repeated three times with six replicates per treatment in each experiment.

\section{Cell apoptosis assay}

This assay was performed using an Annexin V-FITC 
apoptosis detection kit (BD Biosciences, Shanghai, China). According to the manufacturer's instructions, $1 \times 10^{6}$ PaTu8988 cells untreated, or treated with gemcitabine $(8 \mu \mathrm{M})$, Huaier $(6 \mathrm{mg} / \mathrm{mL})$ or gemcitabine $(8 \mu \mathrm{M})$ plus Huaier $(6 \mathrm{mg} / \mathrm{mL})$ were cultured in complete medium for 24 h. After harvested, PaTu8988 cells $\left(1 \times 10^{5}\right.$ cells $)$ were stained with $5 \mu \mathrm{L}$ of propidium iodide (PI) and Annexin $\mathrm{V}$-FITC in $100 \mu \mathrm{L}$ of $1 \times$ Annexin $\mathrm{V}$ binding buffer and incubated in the dark for $15 \mathrm{~min}$ at room temperature. Finally, $400 \mu \mathrm{L}$ of $1 \times$ binding buffer was added and apoptotic cells were analyzed by flow cytometry.

\section{Wound migration assay}

The PaTu8988 cells were seeded in 6-well plates at a density of $5 \times 10^{5}$ cells and cultured to $100 \%$ confluence. The wound was then scratched in the center of the cell monolayer using a $20 \mu \mathrm{L}$ pipette tip. Subsequently, each well was washed 3 times with fresh medium to remove the cell debris and replaced with fresh medium, or the medium containing gemcitabine $(8 \mu \mathrm{M})$, Huaier $(6 \mathrm{mg} / \mathrm{mL})$ or gemcitabine $(8 \mu \mathrm{M})$ plus Huaier $(6 \mathrm{mg} / \mathrm{mL})$. Finally, the wound was photographed under a microscope at an indicated time of 0,24 and $48 \mathrm{~h}$ after the wound was scratched. Would healing areas were calculated and analyzed quantitatively by Image $\mathrm{J}$ software.

\section{Transwell invasion assay}

For the cell invasion assay, Transwell chambers $(8 \mu \mathrm{m}$-pore, Corning, Inc., USA) were coated with $50 \mu \mathrm{L}$ of Matrigel (1:8 pre-diluted with serum-free medium) (Corning, Inc.). Next, $2 \times 10^{5}$ cells which were untreated, or pretreated with gemcitabine $(8 \mu \mathrm{M})$, Huaier $(6 \mathrm{mg} / \mathrm{mL})$, or gemcitabine $(8 \mu \mathrm{M})$ plus Huaier $(6 \mathrm{mg} / \mathrm{mL})$, were added to the upper chamber in a $100 \mu \mathrm{L}$ of serum-free medium. The lower chamber was then supplemented with a $600 \mu \mathrm{L}$ of culture medium. After $48 \mathrm{~h}$, cells which had invaded the lower chamber were fixed with $4 \%$ paraformaldehyde for $15 \mathrm{~min}$ and stained with $0.5 \%$ crystal violet for $20 \mathrm{~min}$ at room temperature. The number of invading cells was counted under a light microscope in at least 6 fields, and the experiment was repeated three times.

\section{Nude mice}

Pathogen-free, 4-week-old, female, athymic BALB/c nude mice (average weight, $15 \mathrm{~g}$ ) were obtained from the Shanghai Laboratory Animal Center (Shanghai, China).
The mice were maintained in specific pathogen-free (SPF) barrier facilities under a $12 \mathrm{~h}$ light/dark cycle, and supplied with sterilized food and water in the animal center of Soochow University. Animal experiments were performed under a project license (A201903036) granted by the ethics committee for research on laboratory animal use of the Soochow University, in compliance with the institution's guidelines for the care and use of laboratory animals.

\section{In vivo animal experiments}

To establish in vivo subcutaneous xenograft models, each mouse was subcutaneously injected with $2 \times 10^{6}$ PaTu8988 cells into the right flank. After mice had generated tumors of approximately 4-5 mm (about 1 week after injection), 12 mice were randomly selected and divided into 4 groups $(n=3$ per group): the untreated group, the gemcitabine-treated group, the Huaier-treated group, and the gemcitabine plus Huaier-treated group. In the untreated control groups, mice were fed with normal saline. In the drug groups, mice were treated with gemcitabine $(1.5 \mathrm{mg} / 0.1 \mathrm{~mL}$ per mouse, twice a week, intraperitoneal injection), Huaier $(50 \mathrm{mg} / 0.1 \mathrm{~mL}$ per mouse, every day, oral gavage) or gemcitabine $(1.5 \mathrm{mg} / 0.1$ $\mathrm{mL}$ per mouse, twice a week, intraperitoneal injection) plus Huaier (50 mg/0.1 mL per mouse, every day, oral gavage), independently. Tumor sizes were measured every week, and the tumor volume was calculated using the following formula: volume $\left(\mathrm{mm}^{3}\right)=$ length $\times$ width ${ }^{2} / 2$. When the subcutaneous tumors reached approximately $1.3 \mathrm{~cm}$ in length (approximately 4 weeks after injection), the tumor-bearing mice were humanely euthanized by continuous inhalation with $30 \% \mathrm{CO}_{2}$ for $5 \mathrm{~min}$. To establish tumor lung metastasis models, mice were also randomly divided into the 4 groups described above ( $\mathrm{n}=3$ per group), and $2 \times 10^{6} \mathrm{PaTu} 8988$ cells were injected into the tail vein of each mouse and administered as above described. Six weeks after injection, the mice were humanely euthanized and the lungs of the xenograft mice were excised and their tissues were removed and photographed. Macroscopically observable metastatic nodules on the surface of each tissue were then counted.

\section{Statistical analysis}

All experiments were carried out with at least 3 replicates. The data are shown as mean $\pm \mathrm{SD}$, and the student's t-test was used for comparisons of differences between 2 independent groups. One-way or two-way repeated measures analysis of variance (ANOVA) was used to analyze 
A

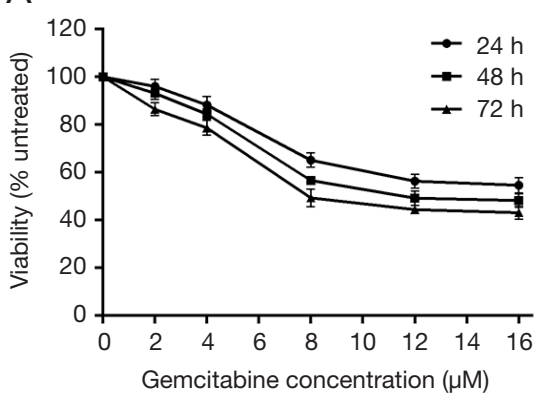

B

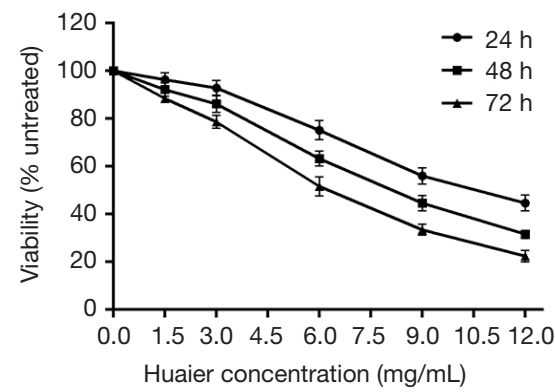

C

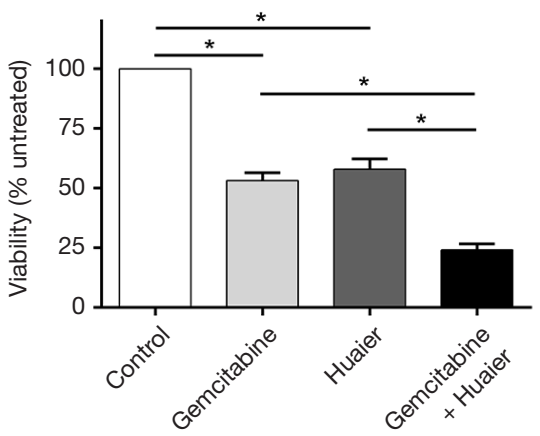

Figure 1 Huaier enhanced the growth inhibition effect of gemcitabine in PaTu8988 cells. A and B: Cell viability analysis of PaTu8988 cells treated with different concentrations of gemcitabine or Huaier at 24, 48, and $72 \mathrm{~h}$ by MTT assay. C: Cells were treated with $8 \mu \mathrm{M}$ of gemcitabine, $6 \mathrm{mg} / \mathrm{mL}$ of Huaier, or a combination of these two drugs- (untreated cells served as a control) for $72 \mathrm{~h}$, with cell viability being measured by MTT assay. *, $\mathrm{P}<0.05, \mathrm{n}=6$ per group, compared with the untreated group or the single drug groups.

the differences among groups, with a $\mathrm{P}$ value $<0.05$ being considered statistically significant. Tests were conducted using SPSS 17.0 (IBM Corp. NY, Armonk, USA).

\section{Results}

\section{Huaier promoted the inbibitory effect of gemcitabine on the growth of pancreatic cancer cells in vitro}

To detect the role of gemcitabine and Huaier in cell viability, we first measured the sensitivity of human pancreatic cancer PaTu8988 cells to gemcitabine and Huaier. We conducted MTT assays to assess the cell viability following exposure to different concentrations of gemcitabine $(0,2,4,8,12$ and $16 \mu \mathrm{M})$ or Huaier $(0,1.5,3.0,6.0,9.0$ and $12.0 \mathrm{mg} / \mathrm{mL})$ at 24, 48 and 72 h. The results are shown in Figure 1A,B, and indicated that both gemcitabine and Huaier inhibited pancreatic cancer cell growth in a time- and dose-dependent manner. It was also revealed that treatment with $8 \mu \mathrm{M}$ gemcitabine or $6 \mathrm{mg} / \mathrm{mL}$ Huaier for $72 \mathrm{~h}$ decreased cell viability by approximately $50 \%$ in $\mathrm{PaTu} 8988$ cells $(\mathrm{P}<0.05)$. Moreover, no increased inhibition effect was observed when cells were exposed to a dose of gemcitabine higher than $8 \mu \mathrm{M}$, showing that pancreatic cancer cells exhibited insensitivity to gemcitabine treatment. To determine the effect of gemcitabine in combination with Huaier on cell growth in vitro, we then treated $\mathrm{PaTu} 8988$ cells with gemcitabine $(8 \mu \mathrm{M})$, Huaier $(6 \mathrm{mg} / \mathrm{mL})$ or gemcitabine $(8 \mu \mathrm{M})$ plus Huaier $(6 \mathrm{mg} / \mathrm{mL})$ for $72 \mathrm{~h}$, and cell viability was again measured by MTT assay. As shown in Figure $1 C$, both gemcitabine and Huaier suppressed PaTu8988 cell growth compared to untreated control cells $(\mathrm{P}<0.05)$. Furthermore, growth inhibition inflicted by gemcitabine plus Huaier treatment was more effective than monotherapy $(\mathrm{P}<0.05)$, indicating that the combination of gemcitabine with Huaier exhibited a synergistic effect on inhibiting pancreatic cancer cell growth. Taken together, our results demonstrated that Huaier promoted the inhibitory effect of gemcitabine on the growth of pancreatic cancer cells in vitro.

\section{Huaier enbanced the apoptotic induction effect of gemcitabine on pancreatic cancer cells}

We next sought to determine whether the inhibition effect of Huaier on cell viability was accompanied by apoptosis induction. The distribution and percentage of cell apoptosis were analyzed in PaTu8988 cells treated with gemcitabine $(8 \mu \mathrm{M})$, Huaier $(6 \mathrm{mg} / \mathrm{mL})$, or gemcitabine $(8 \mu \mathrm{M})$ plus Huaier $(6 \mathrm{mg} / \mathrm{mL})$, and untreated control cells. As shown in Figure 2, gemcitabine or Huaier treatment alone induced PaTu8988 cells apoptosis, with gemcitabine achieving $24.0 \%$ apoptosis, including $11.5 \%$ in early and $12.5 \%$ in late apoptotic cells, and Huaier achieving $11.6 \%$ apoptosis including $7.6 \%$ early and $4 \%$ late apoptotic cells $(\mathrm{P}<0.05)$. Meanwhile, only $1.5 \%$ of cells were apoptotic in the untreated control group, while in the combination group, the percentage of early and late apoptotic cells was $17.0 \%$ and $13.5 \%$, respectively. The results indicated that cell apoptosis was induced by Huaier treatment alone or in combination with gemcitabine $(\mathrm{P}<0.05)$ and that combination treatment markedly induced early apoptosis more effectively than did single drug use $(\mathrm{P}<0.05)$. 
A
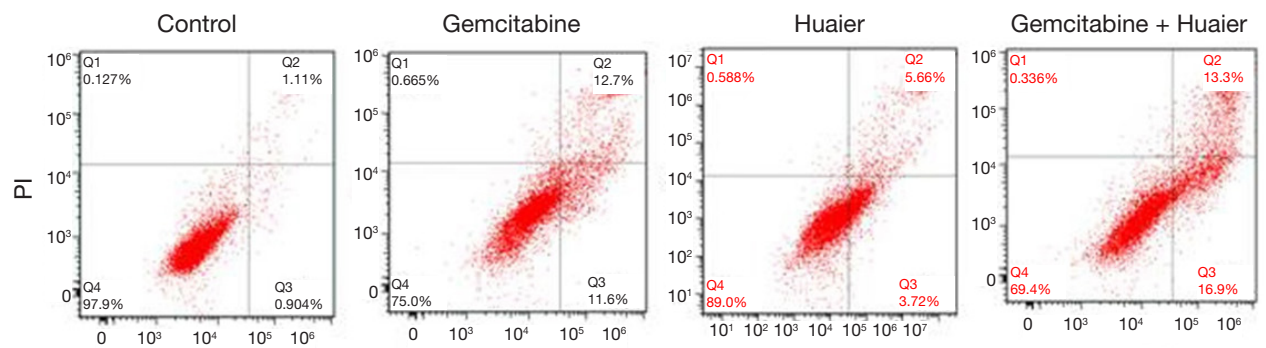

Annexin-V-FITC

B

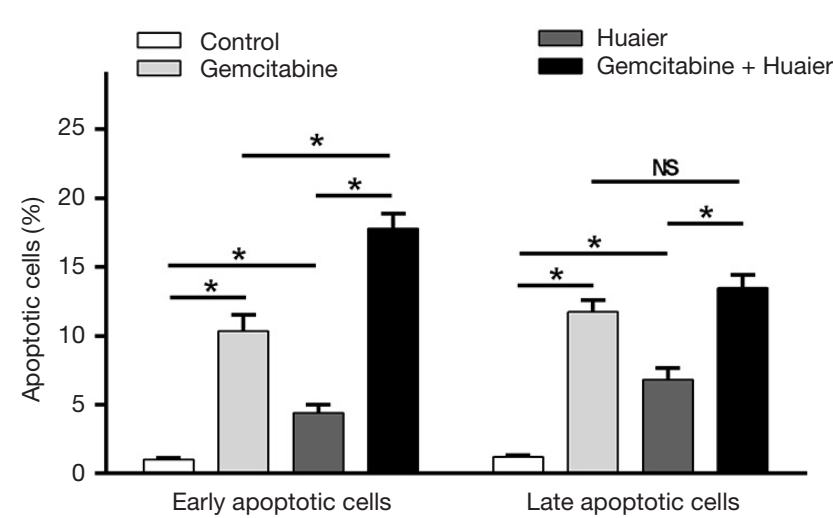

Figure 2 Synergistic effects of gemcitabine combined with Huaier on pancreatic cancer cell apoptosis. Flow cytometric analysis of PaTu8988 cells untreated or treated with gemcitabine $(8 \mu \mathrm{M})$, Huaier $(6 \mathrm{mg} / \mathrm{mL})$ or a combination of these two drugs. (A) Cells were stained with Annexin-V-FITC and analyzed by flow cytometry, with representative flow cytometric images being shown. (B) The percentage of apoptotic cells including early apoptotic cells (annexin V-FITC single-positive cells) and late apoptotic cells (annexin V-FITC/PI double-positive cells) in the total cell population. Compared with the untreated group or single drug groups, ${ }^{*} \mathrm{P}<0.05$; NS, no significance ( $\mathrm{n}=6$ per group).

Although no significant difference was detected in the late apoptotic cells induced by combination treatment compared to gemcitabine treatment (NS, $\mathrm{P}>0.05$ ), gemcitabine and Huaier significantly induced pancreatic cancer cell apoptosis with a synergistic effect (Figure 2).

\section{Huaier promoted the gemcitabine-induced inbibition of pancreatic cancer cell migration and invasion in vitro}

To investigate the effects of gemcitabine and Huaier on the migration and invasion of pancreatic cancer cells, wound migration and Transwell invasion assays were performed in PaTu8988 cells including the untreated control, gemcitabine, Huaier, and combination groups. The results showed that the wound-healing ability of gemcitabine- or Huaier-treated cells was significantly decreased compared to that of the untreated cells $(\mathrm{P}<0.05)$ (Figure $3 A, B)$. In addition, gemcitabine combined with
Huaier significantly improved the efficiency of inhibiting cancer cell migration when compared with single-agent gemcitabine or Huaier $(\mathrm{P}<0.05)$ (Figure $3 A, B)$. The Transwell invasion assay also demonstrated the synergistic role of Huaier and gemcitabine. As shown in Figure 3C,D, the number of invaded cells in the gemcitabine- or Huaieralone treatment groups decreased in comparison to the untreated control group $(\mathrm{P}<0.05)$. Moreover, the invaded cell numbers in the combination group were lower than those of the gemcitabine or Huaier-alone groups $(\mathrm{P}<0.05)$. Taken together, our data suggested that Huaier promoted the suppressing effects of gemcitabine on the migration and invasion of pancreatic cancer cells.

\section{Huaier increased the ability of gemcitabine to suppress tumor growth and metastasis in pancreatic cancer in vivo}

To further determine the synergistic contribution of Huaier 
A

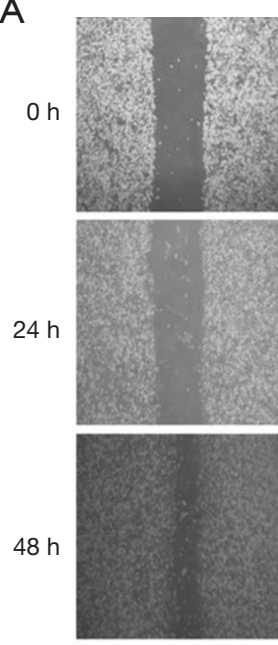

Control
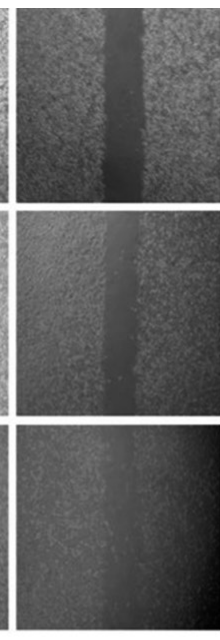

Gemcitabine

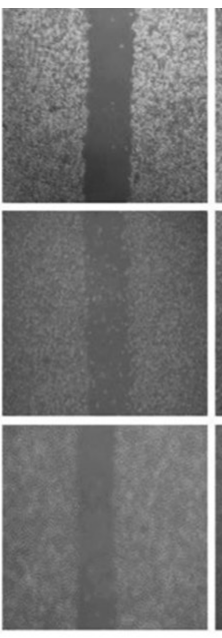

Huaier Gemcitabine + Huaier
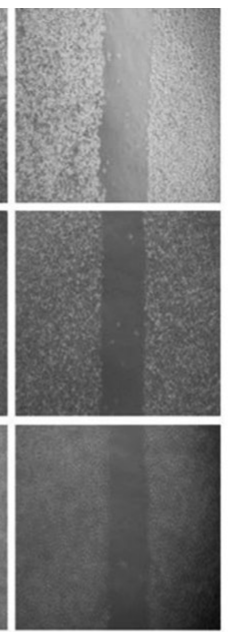

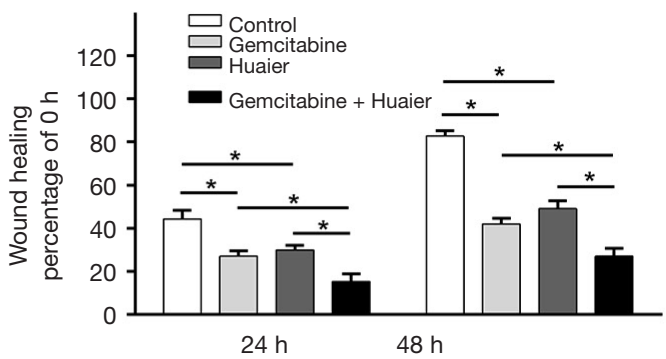

D

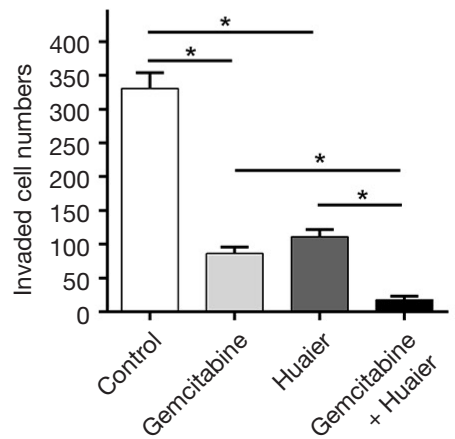

Figure 3 Huaier promoted the suppressing effects of gemcitabine on the migration and invasion of PaTu8988 cells. A and B: The results of wound migration assays in PaTu8988 cells left untreated ortreated with gemcitabine $(8 \mu \mathrm{M})$, Huaier $(6 \mathrm{mg} / \mathrm{mL})$, or their combination. (A) Representative images of would migration assays (magnification, $\times 200$ ). (B) Quantitative analysis of the migration ability of PaTu8988 cells. (C,D) The results of Transwell invasion assays in PaTu8988 cells left untreated or treated with gemcitabine $(8 \mu \mathrm{M}), \mathrm{Huaier}(6 \mathrm{mg} / \mathrm{mL})$, or the combination. (C) Representative Transwell invasion images (crystal violet staining, $\times 200$ ). (D) Quantitative analysis of the effect of drugs on cell invasion. *, $\mathrm{P}<0.05$ ( $\mathrm{n}=6$ per group) compared with the untreated group or single drug groups.

and gemcitabine in the growth and metastasis of pancreatic cancer in vivo, we established subcutaneous xenograft and tail vein injection lung metastasis models using PaTu8988 cells in nude mice. All mice survived for the duration of the treatment. We first studied the influences of gemcitabine and Huaier alone and in combination on the growth of a PaTu8988 pancreatic cancer subcutaneous xenograft, and tumor volume and weight were monitored. As shown Figure $4 A-C$, compared with the untreated control group, tumors treated with either gemcitabine or Huaier alone had lower volumes and weights $(\mathrm{P}<0.05)$. Tumors treated with gemcitabine plus Huaier were also smaller and lighter than those treated with monotherapies $(\mathrm{P}<0.05)$, demonstrating a synergistic reduction effect of gemcitabine and Huaier in pancreatic cancer growth in vivo. We also explored the metastasis of $\mathrm{PaTu} 8988$ pancreatic cancer cells. As displayed in Figure 4D,E, the number of tumor metastatic lung nodules in the gemcitabine or Huaier groups was lower than that of the untreated group $(\mathrm{P}<0.05)$. Compared to treatment with either gemcitabine or Huaier alone, the combination treatment resulted in significantly decreased tumor metastasis in the PaTu8988 pancreatic cancer tail vein injection lung metastasis models $(\mathrm{P}<0.05)$. These data suggested that Huaier enhanced the efficiency of gemcitabine in inhibiting pancreatic cancer growth and metastasis in vivo.

\section{Discussion}

Pancreatic cancer is one of the most malignant tumors and has one of the lowest 5 -year survival rates (1). For patients with the advanced and metastatic form 


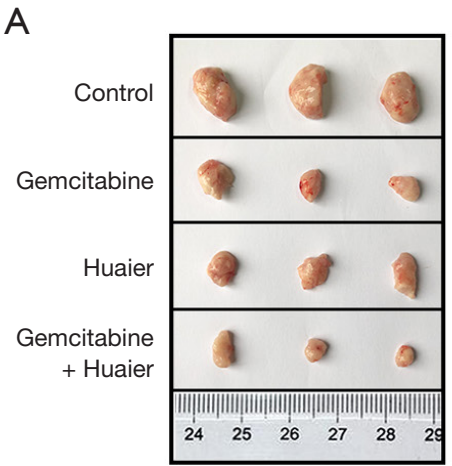

D

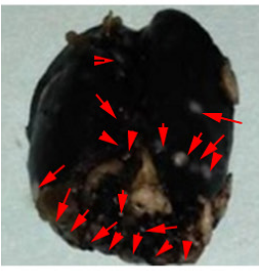

Control

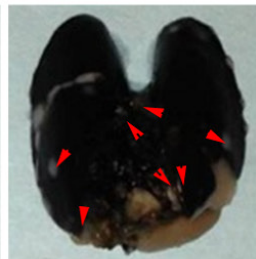

Gemcitabine

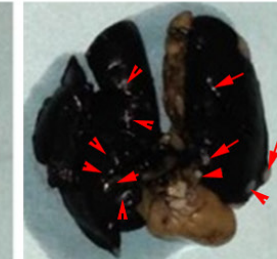

Huaier
B

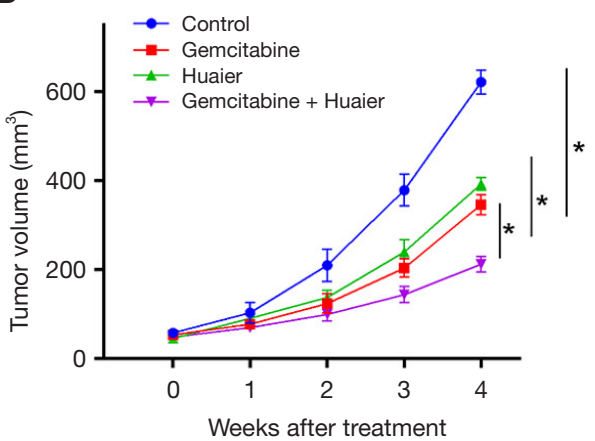

$E$

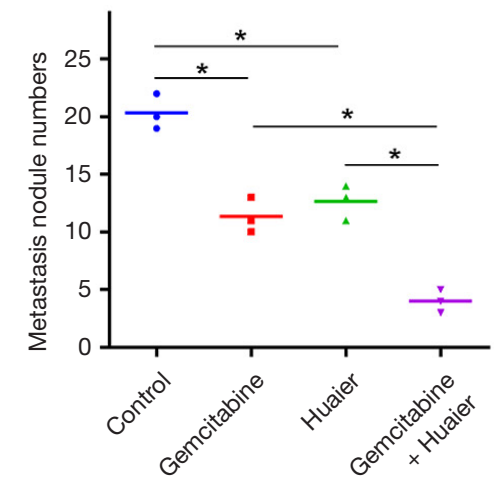

Figure 4 Huaier increased the ability of gemcitabine to inhibit tumor growth and metastasis in subcutaneous xenograft and lung metastasis nude mice models. A and C: The nude mice bearing PaTu8988 subcutaneous xenografted tumors were treated with normal saline, gemcitabine $(1.5 \mathrm{mg} / 0.1 \mathrm{~mL}$, twice a week, intraperitoneal injection), Huaier $(50 \mathrm{mg} / 0.1 \mathrm{~mL}$, every day, oral gavage) or gemcitabine (1.5 $\mathrm{mg} / 0.1 \mathrm{~mL}$, twice a week, intraperitoneal injection) plus Huaier $(50 \mathrm{mg} / 0.1 \mathrm{~mL}$, every day, oral gavage). (A) The images of tumors removed 4 weeks after treatment with drugs. Meanwhile, the tumor volume (B) was measured at indicated times, and the tumor weight (C) was measured 4 weeks after treatment. (D,E) Lung metastatic nodules in PaTu8988 pancreatic cancer tail vein injection lung metastasis models. (D) Representative images of the lungs and nodules removed 6 weeks after treatment with drugs mentioned above (the red arrows, metastatic lung nodules). (E) The number of tumor metastatic nodules in lung tissues. *, $\mathrm{P}<0.05$ ( $\mathrm{n}=3$ per group) compared with the untreated group or single drug treatment group.

of this disease, chemotherapy is the major method for the treatment of patients. As one of the most effective chemotherapy agents for improving the prognosis of cancer patients, gemcitabine has been used as a firstline drug for pancreatic cancer therapy since 1996 (18). Compared with 5-fluorouracil (5-FU), the clinical efficacy response of gemcitabine has shown satisfying outcomes, with a significantly improved median survival rate (19). However, development of chemoresistance to gemcitabine limits its usage in clinical practice (20). Therefore, there is an urgent need to develop novel anticancer agents with fewer side-effects to reduce gemcitabine resistance and enhance the efficacy of chemotherapy, thus satisfying the therapeutic demand of pancreatic cancer patients. Increasingly, studies have focused on the anticancer efficiency of TCM alone or combined with chemotherapy (21-23). The combination of some TCM extracts and gemcitabine are also reported to have better antitumor effects in pancreatic cancer therapy than gemcitabine alone. Oridonin was shown to overcome gemcitabine resistance in PANC-1/Gem cells (24), and melittin, Qingyihuaji formula (QYHJ) and curcumin have been demonstrated to promote gemcitabine sensitivity in human pancreatic cancer treatment (25-27). However, to the best of our knowledge, the anticancer effect of the combination of Huaier and gemcitabine has not been 
reported. Therefore, in this study, we performed in vitro and in vivo studies to explore the antitumor effect of gemcitabine combined with Huaier in pancreatic cancer.

As a type of officinal fungi, Huaier has been used in China for nearly 1,600 years, and has been reported to exert anticancer effects in many cancers (8-17). In accordance with these reports, our studies showed that Huaier alone could reduce cell viability, induce apoptosis, and inhibit the cell migration and invasion ability of pancreatic cancer cells. In vivo animal experiments also demonstrated the effect of Huaier on suppressing tumor growth and lung metastasis in PaTu8988 subcutaneous xenograft and tail vein injection lung metastasis models. These results are consistent with those of a previous study reporting the suppressing role of Huaier in $\mathrm{MiaPaCa}-2$ and Panc-1 pancreatic cancer cells (17).

Several pancreatic cancer cell lines, including PaTu8988, are intrinsically resistant toward gemcitabine (28-30). We performed an MTT assay to assess cell viability following exposure to different concentrations of gemcitabine $(0$, 2, 4, 8, 12 and $16 \mu \mathrm{M})$ or Huaier $(0,1.5,3.0,6.0,9.0$ and $12.0 \mathrm{mg} / \mathrm{mL}$ ) at 24,48 , and $72 \mathrm{~h}$ in PaTu8988 pancreatic cancer cells. In accord with previous studies (28-30), our results also indicated that no increased inhibition effect of cell viability was observed when cells were exposed to a dose of gemcitabine higher than $8 \mu \mathrm{M}$. We also observed that treatment with $8 \mu \mathrm{M}$ gemcitabine or $6 \mathrm{mg} / \mathrm{mL}$ Huaier for $72 \mathrm{~h}$ decreased cell viability by approximately $50 \%$ in PaTu8988 cells. Thus, to identify the suppressing effect of gemcitabine combined with Huaier on pancreatic cancer, $8 \mu \mathrm{M}$ gemcitabine and $6 \mathrm{mg} / \mathrm{mL}$ Huaier were used in later assays. This showed that the combination of gemcitabine and Huaier significantly inhibited the proliferation, migration, and invasion of pancreatic cancer cells compared to either Huaier or gemcitabine monotherapy.

The underlying mechanism for the development of gemcitabine resistance remains unclear. One possible mechanism involves the activation of anti-apoptotic signaling pathways (31). For example, upregulation of Bcl2, Mcl-1, and Bcl-xL are correlated with the increased resistance of pancreatic cancer cells to gemcitabine, while overexpression of $\mathrm{Bax}$ and the high ratio of $\mathrm{Bax} / \mathrm{Bcl}-$ 2 participates in increasing the sensitivity of tumors to gemcitabine (32). Recently, Huaier has been demonstrated to induce apoptosis in numerous cancers (33-35). Zhang et al. (33) verified that Huaier extract causes cell apoptosis and arrests the hepatocellular carcinoma cells in $S$ phase via the JNK pathway. Huaier extract was also found to induce apoptosis in breast cancer and gastric cancer cells (34). Several other researches have focused on the effects of Huaier and chemotherapy drugs such as the synergistic antitumor effect of Huaier combined with 5-FU in human cholangiocarcinoma cells (35), the enhanced efficiency of paclitaxel combined with Huaier extract in breast cancer cell treatment in vitro and in vivo (36), and the potential capacity of Huaier polysaccharide in preventing cisplatin-induced nephrotoxicity (7). However, few studies have investigated the effect of Huaier combined with gemcitabine therapy. In this study, when combined with Huaier, gemcitabine markedly induced cell apoptosis in the PaTu8988 pancreatic cancer cell line, especially the early apoptosis. Although no significant difference was detected in the late apoptotic cells induced by combination treatment compared to gemcitabine treatment alone, the synergistic effect of gemcitabine and Huaier on inducing apoptosis was demonstrated.

Consistent with in vitro results, the in vivo animal experiments also suggested that Huaier could enhance the efficiency of gemcitabine in inhibiting tumor growth and lung metastasis. In subcutaneous xenograft and tail vein injection lung metastasis nude mice models, mice administered with gemcitabine plus Huaier generated smaller and lighter tumors along with fewer lung metastatic nodules compared to single drug treatment. Taken together, these results revealed the synergistic effects of Huaier and gemcitabine in inhibiting pancreatic cancer in vitro and in vivo, indicating that Huaier could enhance the efficiency of gemcitabine in pancreatic cancer therapy. One recent publication has suggested that Huaier might restrain pancreatic cancer by suppressing the $\mathrm{Wnt} / \beta$-catenin pathway (17), which may be an avenue of further research into identifying the underlying mechanisms of Huaier's impact on pancreatic cancer.

\section{Conclusions}

In summary, the present study demonstrated that gemcitabine combined with Huaier exerted a significantly increased effect on inhibiting the proliferation, migration, and invasion of pancreatic cancer cells. In addition, the combination induced in vitro apoptosis and suppressed tumor growth and metastasis in vivo, suggesting that Huaier may act as a novel drug for enhancing the anticancer effect of gemcitabine in pancreatic cancer treatment. However, further studies are warranted to explore the underlying molecular mechanisms for the antitumor effects of Huaier and the roles of Huaier on restraining gemcitabine 
resistance in pancreatic cancer.

\section{Acknowledgments}

We thank the entire staff of the Animal Experimental Center of Soochow University for helpful assistance.

Funding: This study was supported by grants from the National Science Foundation of China (no. 31770985, no. 82073180), Jiangsu Provincial Key Research and Development Program, China (no. BE2019665), Jiangsu Provincial Medical Youth Talent, China (no. QNRC2016732), Jiangsu Provincial "Six Peaks Talent" Program, China (no. 2016-WSW-043), Suzhou Municipal Project of Gusu Health Talent, Young Top Talent, China (no. 2018-057), Gusu Health Talents Cultivation Program, China (no. GSWS2019028), Scientific Research Program of Jiangsu Provincial "333 Talents Projects”, China (no. BRA2019327), Science and Technology Program of Suzhou City, China (no. SYS2019053, no. SLC201906).

\section{Footnote}

Reporting Checklist: The authors have completed the ARRIVE reporting checklist. Available at http://dx.doi. org/10.21037/tcr-20-2627

Data Sharing Statement: Available at http://dx.doi. org/10.21037/tcr-20-2627

Peer Review File: Available at http://dx.doi.org/10.21037/ tcr-20-2627

Conflicts of Interest: All authors have completed the ICMJE uniform disclosure form (available at http://dx.doi. org/10.21037/tcr-20-2627). The authors have no conflicts of interest to declare.

Ethical Statement: The authors are accountable for all aspects of the work in ensuring that questions related to the accuracy or integrity of any part of the work are appropriately investigated and resolved. Animal experiments were performed under a project license (A201903036) granted by the ethics committee for research on laboratory animal use of the Soochow University, in compliance with the institution's guidelines for the care and use of laboratory animals.

Open Access Statement: This is an Open Access article distributed in accordance with the Creative Commons Attribution-NonCommercial-NoDerivs 4.0 International License (CC BY-NC-ND 4.0), which permits the noncommercial replication and distribution of the article with the strict proviso that no changes or edits are made and the original work is properly cited (including links to both the formal publication through the relevant DOI and the license). See: https://creativecommons.org/licenses/by-nc-nd/4.0/.

\section{References}

1. Siegel RL, Miller KD, Jemal A. Cancer statistics, 2020. CA Cancer J Clin 2020;70:7-30.

2. Halbrook CJ, Lyssiotis CA. Employing Metabolism to Improve the Diagnosis and Treatment of Pancreatic Cancer. Cancer Cell 2017;31:5-19.

3. Zhu H, Li T, Du Y, et al. Pancreatic cancer: challenges and opportunities. BMC Med 2018;16:214.

4. Rajabpour A, Rajaei F, Teimoori-Toolabi L. Molecular alterations contributing to pancreatic cancer chemoresistance. Pancreatology 2017;17:310-20.

5. Calabretta S, Bielli P, Passacantilli I, et al. Modulation of PKM alternative splicing by PTBP1 promotes gemcitabine resistance in pancreatic cancer cells. Oncogene. 2016;35:2031-9.

6. Song $\mathrm{X}, \mathrm{Li} \mathrm{Y}$, Zhang $\mathrm{H}$, et al. The anticancer effect of Huaier (Review). Oncol Rep 2015;34:12-21.

7. Fang L, Zhang $\mathrm{Y}$, Wang Q, et al. A polysaccharide from Huaier ameliorates cisplatin nephrotoxicity by decreasing oxidative stress and apoptosis via PI3K/AKT signaling. Int J Biol Macromol 2019;139:932-43.

8. Li Y, Qi W, Song X, et al. Huaier extract suppresses breast cancer via regulating tumor-associated macrophages. Sci Rep 2016;6:20049.

9. Wang W, Wang X, Li C, et al. Huaier Suppresses Breast Cancer Progression via linc00339/miR-4656/CSNK2B Signaling Pathway. Front Oncol 2019;9:1195.

10. Zhang Y, Wang X, Chen T. Efficacy of Huaier granule in patients with breast cancer. Clin Transl Oncol 2019;21:588-95.

11. Li C, $\mathrm{Wu} X$, Zhang $\mathrm{H}$, et al. A Huaier polysaccharide restrains hepatocellular carcinoma growth and metastasis by suppression angiogenesis. Int J Biol Macromol 2015;75:115-20.

12. Shan L, Li Y, Jiang H, et al. Huaier Restrains Proliferative and Migratory Potential of Hepatocellular Carcinoma Cells Partially Through Decreased Yes-Associated Protein 1. J Cancer 2017;8:4087-97. 
13. Zou Y, Xiong H, Xiong H, et al. A polysaccharide from mushroom Huaier retards human hepatocellular carcinoma growth, angiogenesis, and metastasis in nude mice. Tumour Biol 2015;36:2929-36.

14. Chen Y, Wu H, Wang X, et al. Huaier Granule extract inhibit the proliferation and metastasis of lung cancer cells through down-regulation of MTDH, JAK2/STAT3 and MAPK signaling pathways. Biomed Pharmacother 2018;101:311-21.

15. Xie J, Zhuan B, Wang H, et al. Huaier extract suppresses non-small cell lung cancer progression through activating NLRP3-dependent pyroptosis. Anat Rec (Hoboken) 2021;304:291-301.

16. Wei C, Liu Z, Li L, et al. The Anticancer Effect of Huaier Extract in Renal Cancer 786-O Cells. Pharmacology 2018;102:316-23.

17. Zhou C, Li J, Qian W, et al. Huaier extract restrains pancreatic cancer by suppressing Wnt/beta-catenin pathway. Biomed Pharmacother 2020;127:110126.

18. Rothenberg ML, Moore MJ, Cripps MC, et al. A phase II trial of gemcitabine in patients with 5-FU-refractory pancreas cancer. Ann Oncol 1996;7:347-53.

19. Zeng S, Pottler M, Lan B, et al. Chemoresistance in Pancreatic Cancer. Int J Mol Sci 2019;20:4504.

20. Marasini B, Sahu RP. Natural Anti-Cancer Agents: Implications in Gemcitabine-Resistant Pancreatic Cancer Treatment. Mini Rev Med Chem 2017;17:920-7.

21. Ma Z, Fan Y, Wu Y, et al. Traditional Chinese medicinecombination therapies utilizing nanotechnology-based targeted delivery systems: a new strategy for antitumor treatment. Int J Nanomedicine 2019;14:2029-53.

22. Guo Z, Liu Z, Yue H, et al. Beta-elemene increases chemosensitivity to 5-fluorouracil through downregulating microRNA-191 expression in colorectal carcinoma cells. J Cell Biochem 2018;119:7032-9.

23. Yang H, Huang S, Wei Y, et al. Curcumin Enhances the Anticancer Effect Of 5-fluorouracil against Gastric Cancer through Down-Regulation of COX-2 and NF- kappaB Signaling Pathways J Cancer 2017;8:3697-706.

24. Wang B, Shen C, Li Y, et al. Oridonin overcomes the gemcitabine resistant PANC-1/Gem cells by regulating GST pi and LRP/1 ERK/JNK signalling. Onco Targets Ther 2019;12:5751-65.

25. Wang X, Xie J, Lu X, et al. Melittin inhibits tumor growth and decreases resistance to gemcitabine by downregulating cholesterol pathway gene CLU in pancreatic ductal adenocarcinoma. Cancer Lett 2017;399:1-9.
26. Chen P, Wang M, Wang C. Qingyihuaji formula reverses gemcitabine resistant human pancreatic cancer through regulate lncRNA AB209630/miR-373/EphB2-NANOG signals. Biosci Rep 2019;39:BSR20190610.

27. Yoshida K, Toden S, Ravindranathan P, et al. Curcumin sensitizes pancreatic cancer cells to gemcitabine by attenuating PRC2 subunit EZH2, and the lncRNA PVT1 expression. Carcinogenesis 2017;38:1036-46.

28. Jung KH, Choi IK, Lee HS, et al. Oncolytic adenovirus expressing relaxin (YDC002) enhances therapeutic efficacy of gemcitabine against pancreatic cancer. Cancer Lett 2017;396:155-66.

29. Zhao H, Duan Q, Zhang Z, et al. Up-regulation of glycolysis promotes the stemness and EMT phenotypes in gemcitabine-resistant pancreatic cancer cells. J Cell Mol Med 2017;21:2055-67.

30. Zhao X, Zhang GB, Gan WJ, et al. Silencing of B7-H3 increases gemcitabine sensitivity by promoting apoptosis in pancreatic carcinoma. Oncol Lett 2013;5:805-12.

31. Binenbaum Y, Na'ara S, Gil Z. Gemcitabine resistance in pancreatic ductal adenocarcinoma. Drug Resist Updat 2015;23:55-68.

32. Bauer C, Hees C, Sterzik A, et al. Proapoptotic and antiapoptotic proteins of the Bcl-2 family regulate sensitivity of pancreatic cancer cells toward gemcitabine and T-cellmediated cytotoxicity. J Immunother 2015;38:116-26.

33. Zhang C, Zhang J, Li X, et al. Huaier Aqueous Extract Induces Hepatocellular Carcinoma Cells Arrest in S Phase via JNK Signaling Pathway. Evid Based Complement Alternat Med 2015;2015:171356.

34. Qi W, Sun M, Kong X, et al. Huaier extract synergizes with tamoxifen to induce autophagy and apoptosis in ER-positive breast cancer cells. Oncotarget 2016;7:26003-15.

35. Fu Z, Ma K, Dong B, et al. The synergistic antitumor effect of Huaier combined with 5-Florouracil in human cholangiocarcinoma cells. BMC Complement Altern Med 2019;19:203.

36. Yang L, Song Z, Wang X, et al. Huaier extract enhances the treatment efficacy of paclitaxel in breast cancer cells via the NF-kappaB/IkappaBalpha pathway. Oncol Rep 2017;38:3455-64.

Cite this article as: Chen T, Li D, Feng C, Zhang Z, Zhu D, Li D, Zhao X. Huaier increases the antitumor effect of gemcitabine on pancreatic cancer in vitro and in vivo. Transl Cancer Res 2021;10(3):1368-1377. doi: 10.21037/tcr-20-2627 